\section{Use of lipophilic near-infrared dye in whole-body optical imaging of hematopoietic cell homing}

\author{
Vyacheslav Kalchenko, ${ }^{a}$ Shoham Shivtiel, ${ }^{\text {b }}$ \\ Victoria Malina, ${ }^{\text {b }}$ Kfir Lapid, ${ }^{\text {b }}$ Sharon Haramati, \\ Tsvee Lapidot, ${ }^{\text {' }}$ Alexander Brill, ${ }^{c}$ and Alon Harmelin ${ }^{\mathrm{a}, *}$ \\ ${ }^{a}$ Weizmann Institute of Science, Department of Veterinary \\ Resources, Rehovot, Israel 76100 \\ ${ }^{b}$ Weizmann Institute of Science, Department of \\ Immunology, Rehovot, Israel 76100 \\ 'Hadassah Hebrew University Medical Center, Jerusalem, \\ Israel
}

\begin{abstract}
We develop an optical whole-body imaging technique for monitoring normal and leukemic hematopoietic cell homing in vivo. A recently developed nearinfrared (NIR) lipophilic carbocyanine dye 1,1'-dioctadecyl-3, 3, 3', 3'-tetramethylindotricarbocyanine iodide (DiR) is used to safely and directly label the membranes of human leukemic Pre-B ALL G2 cell lines as well as primary murine lymphocytes and erythrocytes. DiR has absorption and fluorescence maxima at 750 and $782 \mathrm{~nm}$, respectively, which corresponds to low light absorption and autofluorescence in living tissues. This allows us to obtain a significant signal with very low background level. A charge-coupled device (CCD)-based imager is used for noninvasive whole-body imaging of DiR-labeled cell homing in intact animals. This powerful technique can potentially visualize any cell type without use of specific antibodies conjugated with NIR fluorescent tag or loading cells with transporter-delivered NIR fluorophores. Thus, in vivo imaging based on NIR lipophilic carbocyanine dyes in combination with advanced optical techniques may serve as a powerful alternative or complementation to other small animal imaging methods. @ 2006 Society of PhotoOptical Instrumentation Engineers. [DOI: 10.1117/1.2364903]
\end{abstract}

Keywords: infrared imaging; fluorescence; biomedical optics; realtime imaging.

Paper 06111LR received May 1, 2006; revised manuscript received Jul. 27, 2006; accepted for publication Aug. 10, 2006; published online Oct. 31, 2006.

Noninvasive, in vivo imaging of targeted cells is a promising field for studying cell behavior and movement in animal models. Intravital microscopy and whole-body fluorescent optical imaging are among the most widely used approaches for visualizing naive or genetically modified cells in vivo. ${ }^{1-3}$ However, advances of fluorescent microscopy are not necessarily applicable for whole-body imaging. For example, fluorescent pigments and reporter genes that have been predominantly used for in vivo imaging emit light in the visible range of spectrum. This is associated with significant limitations,

\footnotetext{
*Tel: 972-8-9344520; E-mail: alon.harmelin@weizmann.ac.il
}

such as tissue light absorption, scattering, and autofluorescence. ${ }^{4}$ In contrast, dyes fluorescing in the near-infrared (NIR) band should be free of this handicap. ${ }^{5}$ To the best of our knowledge, up until now no study has been reported regarding the application of NIR lipophilic dyes for whole-body optical imaging of hematopoietic cell homing.

To study homing patterns of hematopoietic cells, different cell types were utilized: human leukemic Pre-B ALL G2 cells (G2L), primary mouse lymphocytes (ML), and fresh murine red blood cells (RBC). A recently introduced carbocyanine lipophilic NIR fluorescent membrane dye, 1, 1'-dioctadecyl$3,3,3^{\prime}, 3^{\prime}$-tetramethylindotricarbocyanine iodide (DiR, Invitrogen) was used for labeling the cells. ${ }^{6}$ This fluorophore is excited at $750 \mathrm{~nm}$ and has an emission peak at $782 \mathrm{~nm}$. Cells were incubated with $\operatorname{DiR}\left[1 \times 10^{7}\right.$ cells in 10 -ml phosphate buffered saline (PBS) containing $3.5-\mu \mathrm{g} / \mathrm{ml}$ dye and $0.5 \%$ ethanol] for $30 \mathrm{~min}$ at $37^{\circ} \mathrm{C}$. Thereafter, cells were washed twice with PBS and the viability of labeled G2L and ML cells was verified by trypan blue staining. Labeled cells were then injected intravenously into NOD/SCID mice, as well as into CD1 nude mice, which were previously anesthetized by intraperitoneal injection of $35 \mu \mathrm{l}$ of a mixture of ketamine (Rhone Merieux, Lyon, France) and xylazine 2\% (Vitamed, Hedera, Israel) (85:15 vol:vol). Xenogen's IVIS ${ }^{\circledR} 100$ Series Imaging System (Alameda, California) and Olympus SZX12 (Tokyo, Japan) microscope, coupled with a Pixelfly QE (PCO, Kelheim, Germany) charge-coupled device (CCD) camera, were used to monitor DiR labeled G2L and ML localization within live, as well as sacrificed, animals. Imaging was performed at 16-, 24-, and 48-h post cell injection. The excitation and emission filter set in the IVIS was 710 to $760 \mathrm{~nm}$ and 810 to $860 \mathrm{~nm}$, respectively. The emission filter with the wavelength closest to the emission peak of the dye was selected among available filters in the standard configuration of IVIS. An Olympus SZX12 microscope was equipped with a home-built illumination system with a 720- to 750-nm excitation filter and a 780- to 850-nm emission filter, which was placed between the lens and camera. Image processing and data analysis were performed using Living Image 2.5 software and ImageJ version 1.34 (Wayne Rasband, National Institutes of Health, Maryland). All experimental results were repeated on at least three different animals and representative pictures are shown.

To visualize in vivo cell homing, DiR labeled G2L cells or ML were injected intravenously into mice. As depicted in Fig. 1(a), no signal was detected in intact nude mice that had been injected with unlabeled cells. In control experiments, injection of free dye in several concentrations resulted in nonspecific distribution of the fluorescent signal all around the body with no sign of any specific pattern. In animals injected with labeled cells, a general increase of NIR fluorescent signal from the whole body started to develop immediately after injection. During the first $6 \mathrm{~h}$ after injection, a moderate increase in signal intensity was observed in the area of spleen and liver, and homing to specific organs started (data not shown). The fluorescence pattern was clearly detectable in $16 \mathrm{~h}$, reached maximal intensity in $24 \mathrm{~h}$, and persisted up to $48 \mathrm{~h}$. Figures 1 and 2 demonstrate the fluorescent signal at its maximum$24 \mathrm{~h}$ after injection. After $48 \mathrm{~h}$, the signal began to decay,

1083-3668/2006/11(5)/050507/3/\$22.00 @ 2006 SPIE 


\section{JBO LETTERS}
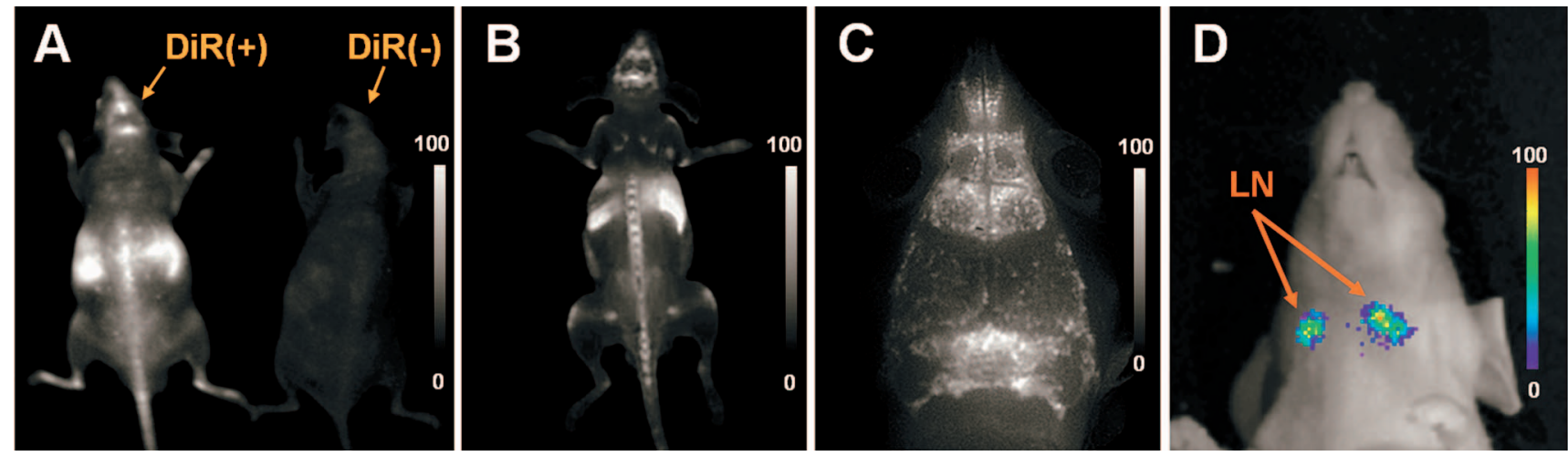

Fig. 1 Near-infrared fluorescent (NIRF) images of DiR labeled cells homing in mice. (a) Noninvasive monochrome NIRF images of unlabeled and DiR labeled G2L cell homing in CD1 nude mice, $24 \mathrm{~h}$ after transplantation of $10^{7}$ cells. $\operatorname{DiR}(+)$ cells were labeled with $\mathrm{DiR}$. DiR(-) cells were not labeled with DiR. (b) Monochrome NIRF image of a sacrificed CD1 nude mouse (skin was removed) $24 \mathrm{~h}$ after transplantation of $10^{7}$ DiR labeled G2L cells. (c) Monochrome NIRF image of the cranium of sacrificed CD1 nude mouse (skin was removed) $24 \mathrm{~h}$ after transplantation of $10^{7}$ DiR labeled G2L cells. (d) Noninvasive color-coded contrast enhanced NIRF image of DiR labeled lymphocyte homing overlaid on a photographic image. LN -superficial cervical lymph nodes of CD1 nude mice $24 \mathrm{~h}$ after transplantation of $10^{7}$ cells. The cutoff of low intensity light was adjusted to make a detected autofluorescent signal below the threshold. Monochrome gradual bar and color gradual bar show intensity of fluorescence in arbitrary units.

remaining detectable although markedly reduced even at 72 to $96 \mathrm{~h}$ (data not shown). In particular, abundant fluorescence was detected in the bone marrow within the spinal column, limbs, and skull. In addition, a strong fluorescent signal was detected in the spleen, liver, and lymph nodes [Figs. 1(a) and 1(d)], reflecting cell accumulation in these specific lymphatic organs. Examination of sacrificed animals after skin removal or isolated organs revealed a stronger signal compared to living mice, and additional regions of previously undistinguishable fluorescence were identified. For example, a weak signal was detected in the scapulae and pelvic bones
[Fig. 1(b)]. In addition, regions in the skull bones infiltrated with labeled cells could be easily distinguished from noninfiltrated regions [Fig. 1(c)]. These findings support previous reports describing lymphocyte homing into the bone marrow and lymphatic organs using other techniques. ${ }^{7,8}$ Thus, it may be concluded that DiR labeling does not impair in vivo homing of human G2L cells and ML.

G2L or ML homing, especially into the bone marrow, is an active process typical only of living cells. Therefore, to distinguish between active homing and passive cell transfer, cells were fixed with $3 \%$ paraformaldehyde in phosphate buffered
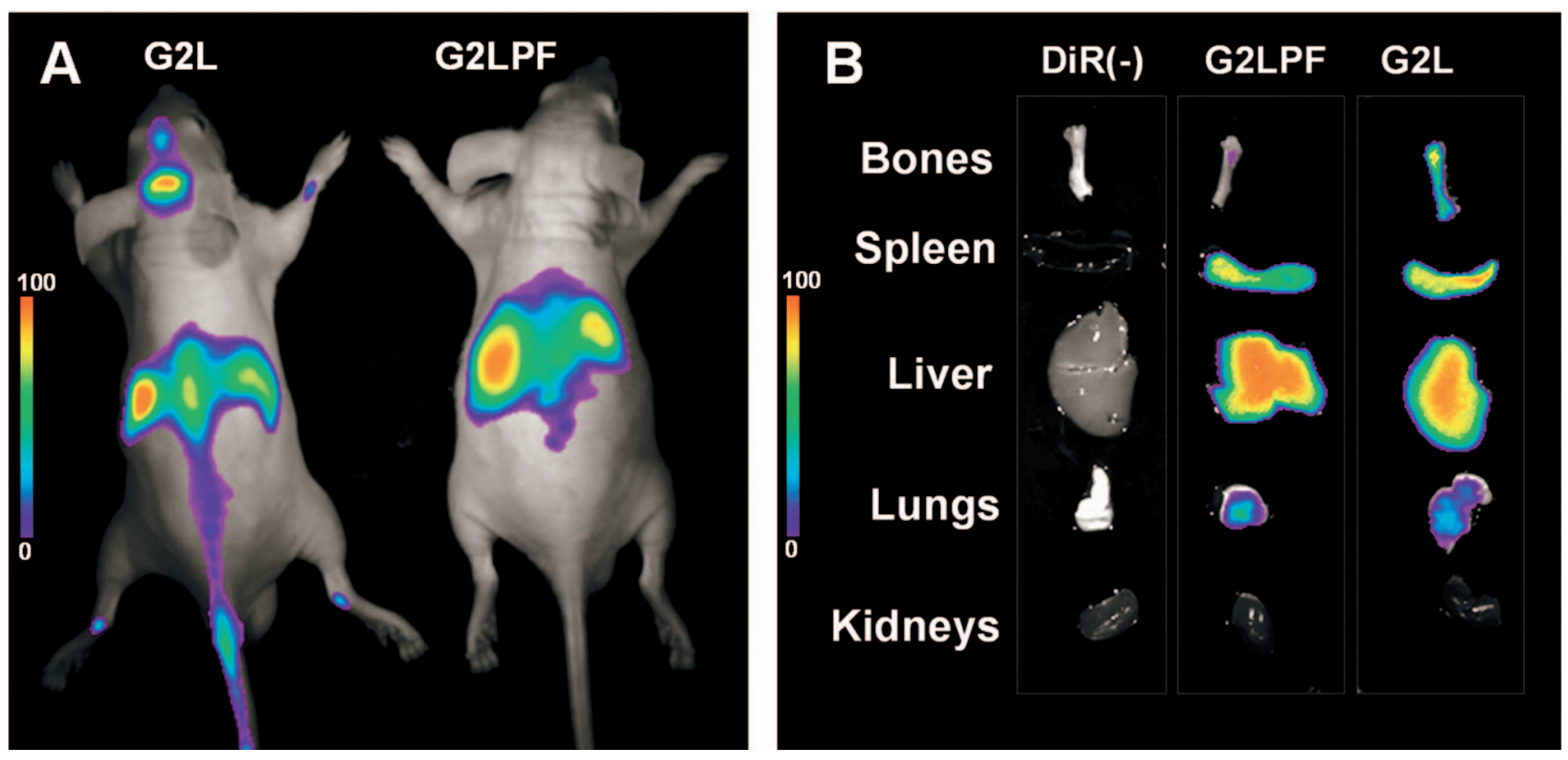

Fig. 2 Effect of paraformaldehyde (PF) on the efficiency of G2L cell homing. (a) Noninvasive color-coded near-infrared fluorescent (NIRF) images of cell homing in CD1 nude mice overlaid on a photographic image $24 \mathrm{~h}$ after transplantation of $10^{7}$ DiR labeled G2L cells (G2L) or fixed DiR labeled G2L cells (G2LPF). (b) Color-coded NIRF image of the internal organs of CD1 nude mice overlaid on a photographic image $24 \mathrm{~h}$ after transplantation of $10^{7} \mathrm{DiR}$ labeled G2L cells. DiR(-) indicate animals injected with G2L cells unlabeled with DiR. The cutoff of low intensity light was adjusted to make a detected autofluorescent signal below the threshold. Color bars show intensity of fluorescence in arbitrary units. 
saline (PBS) for 15 min prior to labeling and injection into mice. Unfixed cells were used as a control. In the experiments in which fixed cells were used, fluorescence was found exclusively in the spleen and liver, suggesting that either dead cells or released dye accumulated in these organs [Fig. 2(a)]. Moreover, a markedly reduced signal, compared to nonfixed DiRlabeled cells, was observed in the bones [Fig. 2(b)]. Notably, no fluorescent signal was detected in the kidneys. This observation suggests that kidneys could serve as a reference organ to which neither G2L nor ML cells home.

Thus, our results for the first time demonstrate that NIR lipophilic carbocyanine dyes can be a powerful tool for both normal and leukemic cell labeling for whole-body optical imaging, providing an efficient alternative to genetic manipulations, staining with other fluorescent reagents, and radioactive cell labeling. Transfection of immune or hematopoietic cells with fluorescent or luminescent reporter genes (for example, luciferase, green fluorescent protein, or red fluorescent protein) prior to their injection is a technically complicated procedure giving relatively low yield that is difficult to standardize. ${ }^{9,10}$ Recently, much attention has been drawn to organic dyes and quantum dots (QDs), NIR light, allowing photons to penetrate up to several centimeters into living tissue. ${ }^{11-13}$ Despite optical advantages of QDs compared to organic dyes, there are still several limitations and concerns regarding the use of QD-bioconjugates for in vivo cellular imaging. ${ }^{14,15}$ A major problem is the difficulty of achieving a high efficiency of labeling during short in-vitro procedures. This is especially critical when hematopoietic cells are utilized, as they are very sensitive to prolonged ex-vivo treatment. In contrast, the proposed method of cellular staining is rapid (15 to $40 \mathrm{~min}$ incubation) and therefore comparatively harmless to cells. Effective labeling of membrane phospholipids by DiR or its analogs in combination with spatial and temporal resolutions (due to optical modality of detection), makes this technique a particularly attractive candidate for in vivo tracking hematopoietic cell movement and homing. This technique can be a cost-effective alternative or complementary approach to other imaging modalities in small animal experimentation.

\section{Acknowledgments}

We thank Ori Brenner and Itai Glinert for helpful discussions and assistance. This work was supported by Teva Pharmaceutical Industries Limited (Young scientists support program).

\section{References}

1. M. Bouvet, J. Wang, S. R. Nardin, R. Nassirpour, M. Yang, E. Baranov, P. Jiang, A. R. Moossa, and R. M. Hoffman, "Real-time optical imaging of primary tumor growth and multiple metastatic events in a pancreatic cancer orthotopic model," Cancer Res. 62, 1534-1540 (2002).

2. M. J. Miller, S. H. Wei, M. D. Cahalan, and I. Parker, "Autonomous T cell trafficking examined in vivo with intravital two-photon microscopy," Proc. Natl. Acad. Sci. U.S.A. 100, 2604-2609 (2003).

3. T. A. Read, M. Farhadi, R. Bjerkvig, B. R. Olsen, A. M. Rokstad, P. C. Huszthy, and P. Vajkoczy, "Intravital microscopy reveals novel antivascular and antitumor effects of endostatin delivered locally by alginate-encapsulated cells," Cancer Res. 61, 6830-6837 (2001).

4. R. Weissleder and V. Ntziachristos, "Shedding light onto live molecular targets," Nat. Med. 9, 123-128 (2003).

5. V. Ntziachristos, C. Bremer, and R. Weissleder, "Fluorescence imaging with near-infrared light: new technological advances that enable in vivo molecular imaging," Eur. Radiol. 13, 195-208 (2003).

6. D. A. Sipkins, X. Wei, J. W. Wu, J. M. Runnels, D. Cote, T. K. Means, A. D. Luster, D. T. Scadden, and C. P. Lin, "In vivo imaging of specialized bone marrow endothelial microdomains for tumour engraftment," Nature (London) 435, 969-973 (2005).

7. P. J. Hendrikx, C. M. Martens, A. Hagenbeek, J. F. Keij, and J. W. Visser, "Homing of fluorescently labeled murine hematopoietic stem cells," Exp. Hematol. 24, 129-140 (1996).

8. S. J. Szilvassy, M. J. Bass, G. Van Zant, and B. Grimes, "Organselective homing defines engraftment kinetics of murine hematopoietic stem cells and is compromised by ex vivo expansion," Blood $\mathbf{9 3}$, 1557-1566 (1999).

9. T. Laitala-Leinonen, "Unsatisfactory gene transfer into boneresorbing osteoclasts with liposomal transfection systems," J. Negat. Results Biomed. 4, 5 (2005).

10. S. Zeamari, G. Rumping, B. Floot, S. Lyons, and F. A. Stewart, "In vivo bioluminescence imaging of locally disseminated colon carcinoma in rats," Br. J. Cancer 90, 1259-1264 (2004).

11. A. P. Alivisatos, W. Gu, and C. Larabell, "Quantum dots as cellular probes," Аnпи. Rev. Biomed. Eng. 7, 55-76 (2005).

12. X. Gao, L. Yang, J. A. Petros, F. F. Marshall, J. W. Simons, and S. $\mathrm{Nie}$, "In vivo molecular and cellular imaging with quantum dots," Curr. Opin. Biotechnol. 16, 63-72 (2005).

13. X. Michalet, F. F. Pinaud, L. A. Bentolila, J. M. Tsay, S. Doose, J. J. Li, G. Sundaresan, A. M. Wu, S. S. Gambhir, and S. Weiss, "Quantum dots for live cells, in vivo imaging, and diagnostics," Science 307, 538-544 (2005).

14. P. Mitchell, "Turning the spotlight on cellular imaging," Nat. Biotechnol. 19, 1013-1017 (2001).

15. S. Ohnishi, S. J. Lomnes, R. G. Laurence, A. Gogbashian, G. Mariani, and J. V. Frangioni, "Organic alternatives to quantum dots for intraoperative near-infrared fluorescent sentinel lymph node mapping," Mol. Imaging 4, 172-181 (2005). 